Editor's Note: These short, critical reviews of recent papers in the Journal, written exclusively by graduate students or postdoctoral fellows, are intended to summarize the important findings of the paper and provide additional insight and commentary. For more information on the format and purpose of the Journal Club, please see http://www.jneurosci.org/misc/ifa_features.shtml.

\title{
Behavioral and Neural Effects of Delays during Intertemporal Choice Are Independent of Probability
}

\author{
Jan B. Engelmann ${ }^{1}$ and Andrew M. Brooks ${ }^{2}$ \\ ${ }^{1}$ Department of Psychiatry and Behavioral Sciences, Emory University School of Medicine, and ${ }^{2}$ Graduate Program in Neuroscience, Emory University, \\ Atlanta, Georgia 30322
}

Review of Luhmann et al. (http://www.jneurosci.org/cgi/content/full/28/53/14459)

Decisions about consequences that are delayed in time are referred to as intertemporal choice. The importance of such decisions can easily be illustrated by their pervasiveness throughout our lives: decisions about education and career, purchasing, saving and investing money, as well as food intake, reproduction and relationships involve tradeoffs between immediate "consumption" and planning for a better future. It is a well established finding in economics and psychology that people tend to prefer immediate over delayed rewards, even if larger sums are offered after a waiting period. For instance, most people would prefer to be paid $\$ 90$ immediately, rather than $\$ 100$ in 2 weeks, a phenomenon referred to as temporal discounting. In an attempt to explain such subjective devaluation of future events, economists and psychologists have modeled discounting behaviors using discount functions such as those illustrated in Figure 1. Although recent studies in neuroeconomics have begun to shed light on the neurobiological basis of subjective valuations during intertemporal choice (McClure et al., 2004; Berns et al., 2006; Kable and Glimcher, 2007), many questions about

Received March 2, 2009; revised March 30, 2009; accepted March 31, 2009. This work was supported by a grant from the National Institute on Drug Abuse to J.B.E. (T32 DA15040).

Correspondence should be addressed to Jan B. Engelmann, 101 Woodruff Circle, Suite 4304, Emory University School of Medicine, Atlanta, GA 30322. E-mail: jan.engelmann@emory.edu.

DOI:10.1523/JNEUROSCI.1037-09.2009

Copyright $\odot$ 2009 Society for Neuroscience $\quad$ 0270-6474/09/296055-03\$15.00/0 the neural mechanisms of intertemporal preferences still remain unanswered.

Intertemporal choice behavior can be influenced by a number of factors, such as the expected value (the product of probability and reward magnitude) of potential outcomes of a choice and, importantly, the length of the waiting period until an outcome is realized. A number of researchers have suggested that delays exert their effect on behavior via enhancing individuals' perceived risk of obtaining a reward (Prelec and Loewenstein, 1991). This explanation relies on the intuition that a large number of unpredictable events may occur between now and the delayed receipt of reward, such as economic downturns that may devalue money, and even death. The question thus remains whether the subjective effects of delays are caused primarily by reducing the perceived probability of an outcome, or whether there are unique aspects inherent to delays that influence intertemporal choice.

A recent study in the Journal of Neuroscience (Luhmann et al., 2008) sought to answer this important question about intertemporal choice by investigating participants' preferences for identical probabilistic monetary rewards that were presented either with or without temporal delays until uncertainty resolution. To do this, the authors developed a novel intertemporal choice task that dissociated delay and probability. Participants, while undergoing functional magnetic reso- nance imaging (fMRI), made dichotomous decisions about financial prospects that varied in amount and probability. Specifically, one option offered the opportunity to win a small cash reward at varying levels of probability, whereas the other option offered twice the amount, but at relatively lower likelihoods. Importantly, decisions were made in the context of two conditions, (1) an immediate and (2) a delay condition. The immediate and delay conditions were similar in that the likelihood of obtaining financial reward was represented visually by the number of rectangles displayed below each option, with an increasing rectangle number reflecting a decrease in likelihood. Importantly, the delay condition differed in that rectangle number also determined the length of the delay until the outcome was revealed, while this occurred immediately after response in the immediate condition. Trial durations were kept constant across the two conditions, so that participants could not simply speed up the experiment by choosing the smaller reward.

An analysis of the behavioral effects of probability and delay on participants' choice proportions revealed two interesting results: (1) choices were influenced by probability, such that participants chose the smaller reward more often as probability of the larger reward decreased relative to that of the smaller reward, and, importantly, (2) choices were influenced by the delay, such that participants chose the larger, less probable reward less frequently 
in the delay condition than in the immediate condition. Of note, the latter effect was observed despite identical probability distributions and reward magnitudes being offered in immediate and delay conditions, indicating that this effect is caused by the delay alone. This finding is important, because it suggests that simply delaying the resolution of uncertainty about consequences of decisions is perceived as aversive by most participants, potentially by increasing anxiety during the delay period as suggested previously ( $\mathrm{Wu}, 1999)$.

These behavioral results were paralleled by the neuroimaging results, which revealed differential activation patterns during the deliberation phase when participants made choices in the delay compared with the immediate condition. The authors used parametric analyses to probe for brain regions whose activity shows correlations with (1) expected value and (2) rectangle number of the chosen option on each trial. Parametric analysis differs from standard fMRI analysis in that it identifies voxels whose activity shows a linear relationship with a continuous variable of interest, e.g., rectangle number or expected value, by predicting the magnitude of hemodynamic responses based on changes in the size of the underlying variable on a trial-by-trial basis. This type of analysis extracts the strength of the relationship between such a continuous variable of interest and activity in a given brain region. Of note are the authors' findings related to rectangle number associated with participants' choices on each trial, which probe for brain regions whose activity shows significant correlations with (1) probability alone in the immediate condition, and (2) probability plus delay in the delay condition. Whereas the authors report no significant correlations with rectangle number in the immediate condition, they demonstrated positive correlations between rectangle number and neural activity in the delay condition in bilateral parahippocampal gyri, anterior cingulate cortex, right superior parietal lobe [Luhmann et al. (2008), their Fig. 4 , top], and right intraparietal sulcus. To further investigate the nature of these differential activation patterns as a function of delay, the authors conducted region of interest (ROI) analyses. These revealed greater correlations in the delay compared with the immediate condition, as reflected by greater regression coefficients, which in the context of parametric modulation represent the slope of the correlation between hemodynamic responses and number of rectangles [Luhmann et al. (2008),

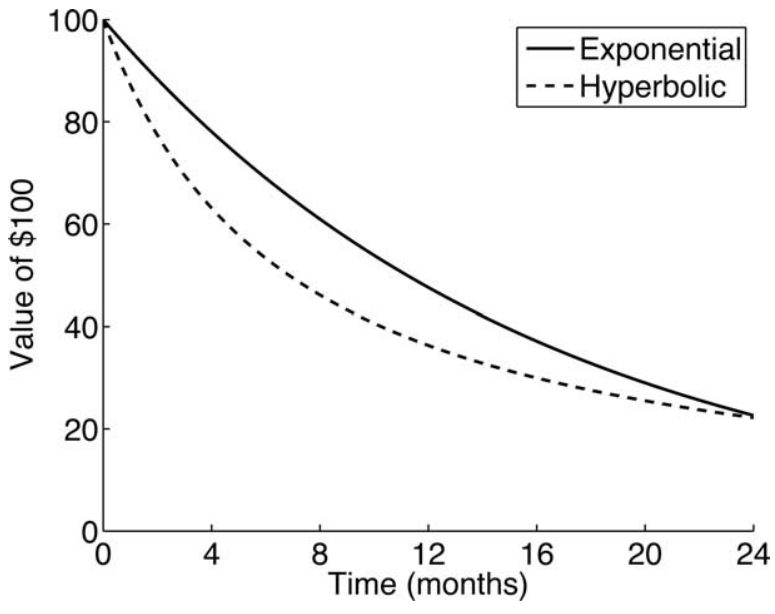

Figure 1. Illustration of discounting functions commonly used to model intertemporal choice behavior (modified from Berns et al., 2007). Discounted utility, the standard model of intertemporal choice in economics, assumes that people discount future gains and losses in an exponential manner (solid line, functional form: $\delta^{t}$, where $t$ represents time and $\delta$ is the discount rate, here $\delta=$ 0.94). Hyperbolic discounting (dotted line) has been demonstrated to provide a better fit to most empirical data for animals and humans [functional form: $1 /\left(k^{*} t+1\right)$, here $\left.k=0.15\right]$. Both models demonstrate that the value of monetary incentives decreases with increasing delay until receipt of reward. For illustration purposes, discount rates are exaggerated. Typical discount rates observed in behavioral economics range between 10 and $25 \%$ over a 12 month period but can be larger depending on context.

their Fig. 4, bottom]. The fact that activity in these regions showed significant parametric modulation by rectangle number only in the delay condition underlines their involvement in the purely temporal aspects of intertemporal choice.

The authors furthermore observed an intriguing heterogeneity in participants' sensitivity to the delay. Previous research in neuroeconomics has demonstrated that individual differences at the behavioral level can have powerful modulatory effects on brain activations (Berns et al., 2006). Participants in Luhmann et al. (2008) exhibited varying attitudes to the delay, ranging along a behavioral continuum demonstrating aversion to the delay (larger reward chosen less frequently in delay compared with immediate condition) to delay indifference (larger reward chosen with equal frequency in delay condition). To capture these individual differences in delay sensitivity, the authors computed difference scores between the probability of choosing the larger reward in delay and immediate conditions for each participant. The authors then correlated behavioral delay sensitivity with its neural equivalent, the difference between activation during the delay and immediate conditions. Such investigations of individual differences are important for a number of reasons: (1) past research in the field of economics has largely ignored these, (2) they can reveal important patterns in the data that may be washed out by standard fMRI analyses, and (3) they directly link behavior to brain activations.
Significant correlations between behavioral and neural sensitivity to the delay were obtained in frontopolar cortex [Luhmann et al. (2008), their Fig. 5]. In particular, the authors' findings indicate a negative relationship between neural activity in frontopolar cortex and behavioral delay sensitivity. This suggests that participants whose choices were significantly influenced by the delay (greater delay sensitivity) recruited the frontopolar cortex more actively. Together with previous findings implicating the frontopolar cortex in representing future events (Buckner and Carroll, 2007), results from Luhmann et al. are consistent with the notion that delays exert their effect on intertemporal choice via people's ability to successfully construct representations of future events, a process referred to as prospection.

In summary, the study by Luhmann et al. (2008) advances research on intertemporal choice by providing evidence for the notion that unique aspects inherent to temporal delays can significantly affect behavior, and, in parallel manner, activation in a network of structures associated with self-referential states. Importantly, these effects of the delay were independent of probability in the context of the task used by Luhmann et al. (2008). The authors interpret their results, showing modulation of activity attributable to temporal aspects of the task in regions previously associated with self-projection and prospection, as suggesting that one important aspect of intertemporal decision-making involves deliberations 
about one's future self. This notion is both interesting and plausible, but the extent and nature of the relationship between prospection and temporal discounting, which was not directly investigated by Luhmann et al. (2008), needs to be addressed by future research. Finally, it will be interesting to see whether results from Luhmann et al. (2008) can be replicated by studies investigating decisions involving extended time intervals ( $>1$ month) (Fig. 1). The task of prospection becomes more complex in such scenarios, and people might rely on choice heuristics or recruit affective processes to guide their decisions when facing such increased complexity. It is therefore likely that inter- temporal decision-making about consequences that are significantly delayed in time will lead to greater recruitment of affective centers, such as the anterior insula and amygdala, which, somewhat surprisingly, were not modulated by temporal aspects in the study by Luhmann et al. (2008). This conjecture, however, remains to be tested by future research.

\section{References}

Berns GS, Chappelow J, Cekic M, Zink CF, Pagnoni G, Martin-Skurski ME (2006) Neurobiological substrates of dread. Science 312:754-758.

Berns GS, Laibson D, Loewenstein G (2007) Intertemporal choice-toward an integrative framework. Trends Cogn Sci 11:482-488.
Buckner RL, Carroll DC (2007) Self-projection and the brain. Trends Cogn Sci 11:49-57.

Kable JW, Glimcher PW (2007) The neural correlates of subjective value during intertemporal choice. Nat Neurosci 10:1625-1633.

Luhmann CC, Chun MM, Yi DJ, Lee D, Wang XJ (2008) Neural dissociation of delay and uncertainty in intertemporal choice. J Neurosci 28:14459-14466.

McClure SM, Laibson DI, Loewenstein G, Cohen JD (2004) Separate neural systems value immediate and delayed monetary rewards. Science 306:503-507.

Prelec D, Loewenstein G (1991) Decision making over time and under uncertainty: a common approach. Manage Sci 37:770-786.

Wu G (1999) Anxiety and decision making with delayed resolution of uncertainty. Theory Decis $46: 159-199$. 